\title{
Roll-to-Roll Compatible Methods and Outlook for Perovskite Solar Cells
}

\author{
Li Chenglin* \\ Northwestern Polytechnical University, School of material science and engineering, Xi'an, China, 710129
}

\begin{abstract}
Perovskite Solar Cells (PSCs) are regarded as a highly effective low-cost solar energy collector, which is promised to sustain considerable energy supplies for modern society. Roll-to-Roll (R2R) compatible PSCs fabrication method is promised to realized industrialized PSCs production. This paper presents an overview of recent R2R compatible methods for PSC fabrication, which covers five R2R compatible strategies towards industrialized R2R production. To further tackle technical obstacles towards the PSC industrialization, improving perovskite morphology, adopting ambient production conditions and enhancing moisture resistance, as three aspects towards a high-effective, low-cost and highly stable PSCs, are discussed and concluded. This paper also discussed the possibility of hybrid wind-solar energy collector and raises a wind-flutter-PSCs composed conceptional structure.
\end{abstract}

\section{Introduction}

Energy sustainability is regarded as a universal concern. Massive energy consumption based on the exhaustion of non-renewable energy sources is unsustainable, while solar energy, as a clean, renewable and sustainable energy source, is promising to maintain energy sustainability in modern society. PSCs have great potential in the effective solar-energy harvest and low-cost production [1,2], which is deemed as a solution to supply massive energy consumption.
PSCs have shown advantages in flexibility and mobility, high defects tolerance and high power-conversion efficiency (PCE) in collecting solar energy $[3,4]$. To achieve extensive application, PSCs should initially have sufficient PCE for energy harvest, as well as industrial methods for industrial production. For the first requirement, laboratory-fabricated PSCs can reach $25.5 \%$ in 2020 , recorded by National Renewable Energy Laboratory (NREL), seeing figure 1[5]. Current PSCs, which are fabricated in laboratory methods, have achieved comparable PCE to Si solar cells. This indicates the approaching trend of PSCs towards commercialized solar cells.



Fig 1. The Best laboratory-fabricated Solar-Cell Efficiencies[5]

\footnotetext{
Corresponding author: chenglin_li_nwpu@163.com
} 
However, methods for industrial manufacture of stable PSCs have been major concern towards the commercialization of PSCs. Currently, the R2R method for PSCs fabrications is well-studied for industrial production of PSCs, and sub-methods within R2R principles have shown diversities in manufacturing technology, product quality and PCE of the cell. Based on existing laboratorial experiments on R2R method for PSCs, the paper will present a review on R2R compatible method and an outlook towards industrialized PSCs productions and a novel application.

\section{Roll-to-Roll method for the fabrication of $n$-i-p perovskite solar cells}

Common n-i-p perovskite solar cells consist of electron transport layers, mesoporous metal oxide layers, perovskite layers, hole transport layers and metal electrodes. Metal oxide is adopted as typically material for mesoporous oxide layers, such as $\mathrm{TiO} 2[1]$ or $\mathrm{ZnO}$ [2]. Perovskite material, as the key component of PSCs, plays a decisive role in deciding the PCE of the cells. Electron-hole pairs are knocked apart by the photons on the perovskite layer, which is crucial to the power conversion from optical energy to electric energy. Certain organic materials, for example, Spiro-OMeTAD or poly (3,4-ethylenedioxythiophene) / poly (styrenesulfonate) (PEDOT:PSS), is adopted as effective hole transport material to conduct hole carriers on PSCs [6,7]. Jeong et al. introduced Spiro-mF and Spiro-oF as hole transport material, which obtains an advancing PCE of $24.8 \%$ [8]. Metal electrodes on PSCs serve as electron conductors to carry drifting electrons in an electric field, which forms electric current.

The R2R fabrication strategy shows strong applicability on the fabrication of n-i-p structure PSCs. The mesoporous oxide precursor, perovskite precursor and hole transport material can be coated onto the conductive substrates in sequence, which can be achieved in a flow line production. The flow line productions to fabricate PSCs can effectively reduce the cost of preparation and raise the efficiency of the preparation techniques, compared to chemical vapor deposition methods or solution methods. In that, the R2R method is currently one of the most adaptable methods for industrial production.

\section{Roll-to-roll compatible fabrication method}

Current R2R method awaits improvements towards large-scale fabrication of high-quality PSCs, and laboratorial R2R compatible methods have been conducted to improve the quality of the PSCs and preparation technics.

\subsection{Roll-to-roll gravure-printing compatible method}

The R2R gravure-printing compatible method enables cost-effective and high-speed production of large-area PSCs, which is applied in laboratorial scalable productions of PSCs. During the R2R gravure print, engraved cylinders were installed to transfer PSC precursor solution onto a flexible substrate. The installed cylinders were partly immerged by the precursor solution, which was infiltrated and can eventually transfer the precursor. The coated substrate is pulled into a further drying process for perovskite crystallizing.

Kim et al. employed gravure printing method to print PSCs with $p-i-n$ structure. Perovskite precursor solution was prepared in an ink container. To further improved the quality of perovskite layer, Kim et al. employed one-step method for the crystallization process, during which the coated perovskite layer was placed in an anti-solvent bath. The proposed all-printed PSCs have reached a high PCE up to $17.2 \%$. Based on the gravure printing method, a R2R gravure printing compatible method show its promising future for its advantageous production speed and cost. Kim et al. demonstrated a R2R gravure printing strategy in laboratorial-scale PSCs production, in which the best PCE recorded reached $11.0 \%$, as shown in figure 2 [9].

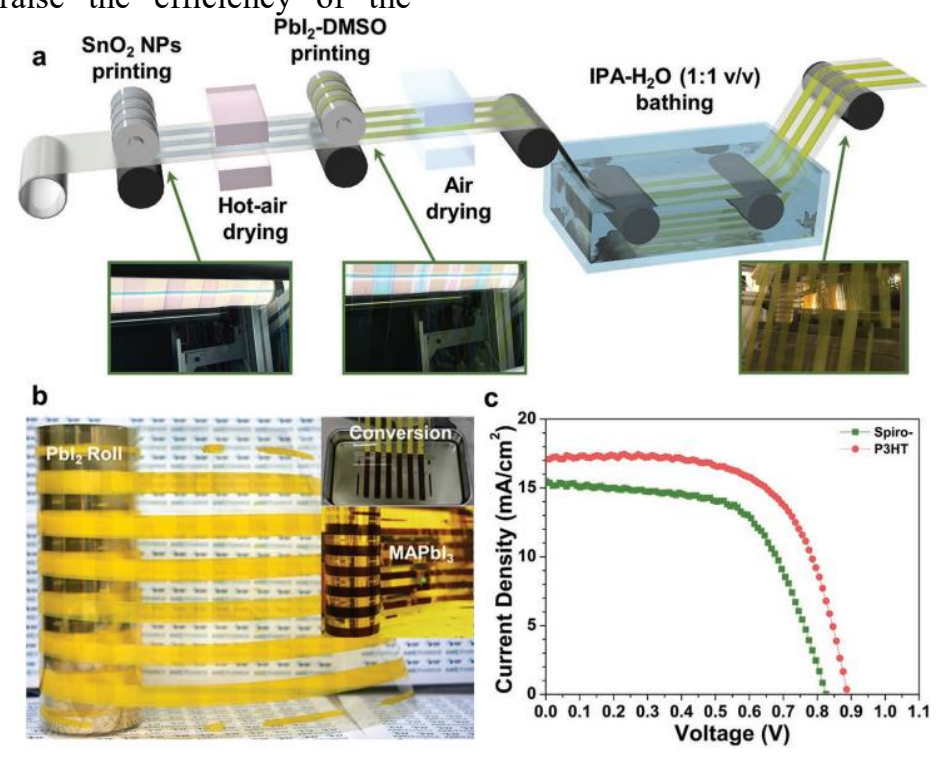

Fig 2. Illustration of R2R Gravure-Printing Compatible Method [9] 
Relatively limited PCEs restricted the commercialization of current R2R gravure printed PSCs. However, considering current best-performed PSCs had reached a PCE of $25.5 \%[5]$, the prospect for the improvements of the PCE of R2R printed PSCs is promising and achievable.

\subsection{Roll-to-Roll Slot-Die Coating Compatible Method}

The slot-die coating is a printing technique, which requires printer to achieve layer deposition of perovskite layer[3]. The R2R method is finely adaptable to layer coating that is required in the slot-die coating PSCs manufacture process, and R2R slot-die compatible method has shown more merits in large-scale PSCs production. The R2R slot-die coating compatible method is classified as pre-meter coating method, which requires precisely controlled parameters. In this way, R2R slot-die coating compatible method can achieve precisely-controlled perovskite layer thickness and enable well-uniformed perovskite layer and high-quality $\operatorname{PSCs}[10]$.
Hwang et al. combined the slot-die coating method and gas-quenching process based on a self-developed printer to fabricate fully slot-die coated PSCs. $\mathrm{CH} 3 \mathrm{NH} 3 \mathrm{PbI} 3$ perovskite layer was coated by a slot-die coating head, which was then followed by a high-press nitrogen drying process, and eventually formed on the electron transporting layer. Nitrogen gas quenching process was adopted to form a cloudy well-uniformed methylammonium iodide (MAI) layer. To further achieve large-scale R2R productions, a fully slot-die coating method was adopted. The best PCE of the target PSC by the R2R slot-die compatible method reached up to $11.96 \%[10]$.

The R2R compatible method has a promising future in manufacturing flexible PSCs device. Sear et al. studied the R2R slot-die coating method on flexible PSC fabrication, as figure 3 Illustrated. $\mathrm{Ag}$ nanowire/PEDOT:PSS electrodes were formed on a PET substrate in substitute for ITO conducting substrate. The Ag nanowire-PET based conducting material was studied to have excellent bending performance compared to traditional ITO glass substrate. The highest PCE of the proposed flexible PSCs reached 11\% [11].

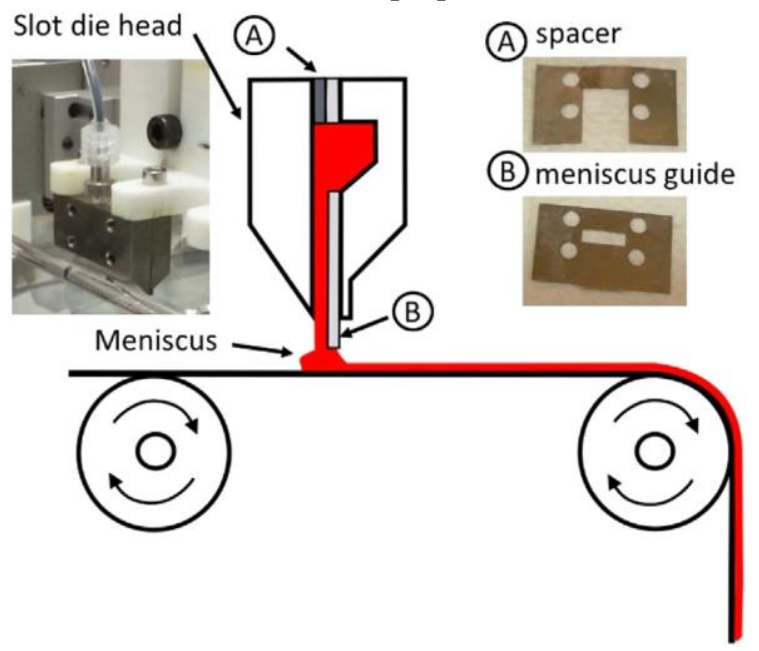

Fig 3. Illustration of R2R slot-die coating compatible method [11]

Kim et al. proposed a shielding-gas-less R2R slot-die process to further achieve a low-cost R2R fabrication, shown in figure 4. The fabrication process was conducted under natural ambient conditions. A high deposition temperature was employed to shorten the processing time exposed to ambient moisture, as illuminated in figure 4(a). Without shielding gas blowing, the heating-only evaporated PSC showed better performance at a higher temperature within a permitted temperature range, which was $130^{\circ} \mathrm{C}$. At the optimized processing temperature, a PCE of $11.62 \%$ was observed [12].

The R2R slot-die coating method has been successfully applied to the fabrication of high-quality, flexible PSC devices. Furthermore, R2R slot-die coating method was improved in processing resistance to ambient moisture. Based on these method advances, a humidity-resisted R2R slot-die coating method for low-cost industrial production was promising.

\subsection{Roll-to-roll doctor-blade coating compatible method}

Doctor-blade coating is a large-scale PSCs fabrication method with low cost and high simplicity, which was applied to the deposition of perovskite thin films and PSC layers. During the doctor-blade coating process, perovskite precursor, which has been transferred onto the substrate beforehand, was swept and spread by a blade. The precursor film was then heated and evaporated, which forms the perovskite layer[13]. The film thickness and quality of the layer are highly influenced by the blade parameters, such as the gap height between the blade and substrate, the sintering temperature and the coating speed. 

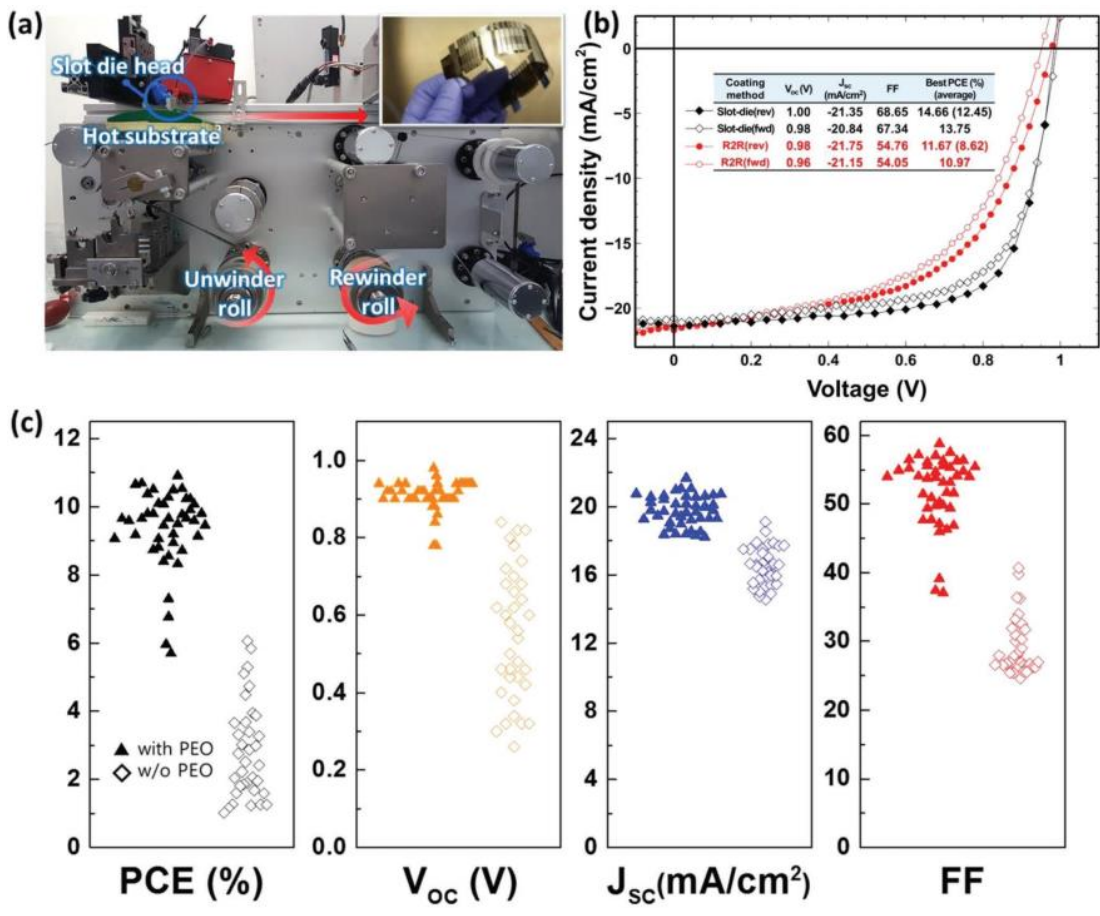

Fig 4. High processing temperature R2R slot-die coating method[12]

The blades can continuously coat the precursor solution to form a constant film-thickness layer, which is highly compatible with R2R method. The pre-dripped perovskite precursor is uniformly doctor-bladed as the $\mathrm{R} 2 \mathrm{R}$ transmission pulls the substrate with the precursor underneath the blade, and $\mathrm{R} 2 \mathrm{R}$ doctor-bladed perovskite layer is formed, which indicates the applicable prospect of $\mathrm{R} 2 \mathrm{R}$ doctor-coating techniques.

Tang et al. adopt doctor-blade coating in preparing MA0.6FA0.38Cs0.02PbI2.975Br0.025 films. A perovskite precursor was mixed with a small proportion of $\mathrm{Cs}+$ cations and $\mathrm{Br}$ - anions to improve the phase purity of perovskite thin film, which also allowed a deposition temperature of $120{ }^{\circ} \mathrm{C}$. Methylammonium chloride $(\mathrm{MACl})$ addition was applied to the mixture to intrigue a secondary grain growth, which resulted in large grain growth. A pin-hole free and high-purity PSCs was obtained, whose PCE reached 19.3\%[14].

The R2R doctor-blading compatible method shows great potential in high-quality PSCs. The perovskite layer of laboratorial PSC by R2R doctor-blading shows a pin-hole free perovskite morphology. Moreover, larger crystalline grains can be obtained compared to spin-coating perovskite films[14], which results in higher stability and performance of R2R doctor-bladed PSCs.

\subsection{Roll-to-roll inject printing compatible method}

Inject-printing method is a highly digital-programmed method which allows precise parameter control over a inject printer. Inject-printing method shows the advantages of precise and time-efficient printing upon small-scale printing[15]. Newsletter on an OLED ink-printing technology reported that an inject-printed organic layer showed less stray particles, which leads to high-quality small-area film[16].
Inject printing was firstly applied to the fabrication of perovskite in 2014 when Wei et al. adopted the printing method to deposit a $\mathrm{CH} 3 \mathrm{NH} 3 \mathrm{PbI} 3 / \mathrm{C}$ bilayer onto the substrates. Carbon-based perovskite material was chosen for its adaptability with printing technology. Perovskite precursor is prepared and filled in the printer, which will print the precursor onto the substrate by printing programming instructions. The proposed inject-printed perovskite solar cells have shown an improved efficiency up to $11.60 \%[17]$.

Inject printing compatible with $\mathrm{R} 2 \mathrm{R}$ strategy combines the advantages of digital program control and large-scale production, which is a highly-automatic and programmable method for effective industrial production. Common R2R production requires flexible substrates. Inject printing technique can easily deposit perovskite precursor onto the substrate of the same principle of typography. Furthermore, the inject-printing method owns high prospects in the manufacture of flexible devices, such as flexible PSCs, perovskite photo detector[6,18] or flexible perovskite quantum dots[19].

However, the current R2R inject-printing method still meets gaps in its realization. It is reported that current large-scale printing shows a lower deposition density than substituted method. Certain technical obstacles await solutions to realize an industrial $\mathrm{R} 2 \mathrm{R}$ inject-printing production[16].

\subsection{Roll-to-roll intense pulsed light compatible method}

The intense pulsed light method is initially applied in industrial semiconductor devices production, which realizes a non-contact light-heat sintering and annealing process. In PSCs productions, the intense pulsed light owns a high photon density over a radiant area. In that, 
adequate light energy can be transmitted to a radiated precursor within a short period, which realizes short-term high temperature drying process. The perovskite layer shows a short processing period, which limits the exposure of perovskite material to ambient moisture, large crystalline grains and well-uniformed morphology, which is demonstrated in figure 5[20].

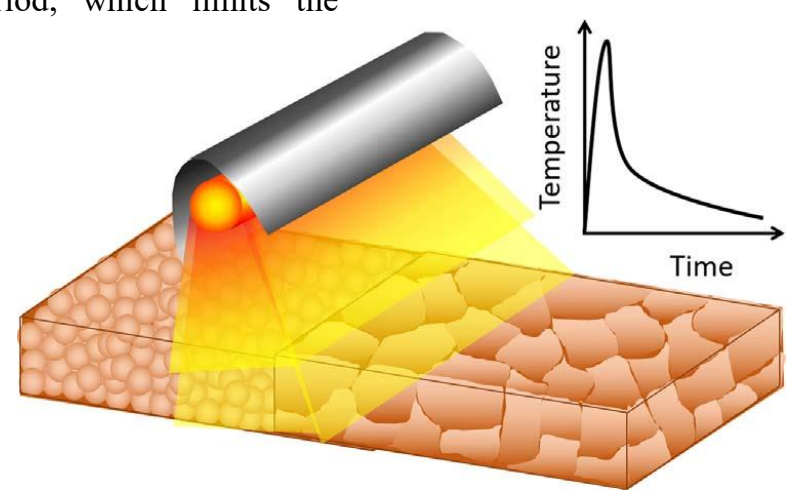

Fig 5. Schematic of crystalline growth after intense pulsed light treatment[20]

Amir H. Ghahremani, and Thad Druffel applied an intense pulsed light method for the $\mathrm{SnO} 2$ electron transport layer fabrication and perovskite thin film annealing. SnO2+ film was sintered under the condition of 5 pulses with $2100 \mathrm{~J}$ energy each pulse. Perovskite layer was annealed under the condition of 5 pulses with $1400 \mathrm{~J}$ energy each. And the annealing processes were conducted in the ambient with a relative humidity of $60 \%$. $\mathrm{Cs}+/ \mathrm{CH} 3 \mathrm{NH} 3+/ \mathrm{CH}(\mathrm{NH} 2) 2+$ triple cation perovskite films with $\mathrm{SnO} 2$ electron transport layer were obtained and the optimal PCE reaches $11.34 \%$ [20].

It was shown that the intense pulsed light method takes a short dying period, which enhances the fabrication resistance to moisture and enables ambient-condition processing. Compared to the high-temperature drying process, the intense pulsed light method can realize short-time dying without damaging the inner layer and the substrates[21]. Kim et al. have raised the problem of the damage of high-temperature to the plastic substrates. and anti-solution drying process[12]. For the intense pulsed light method, high light energy is absorbed by the precursor layer, with limited energy penetrating the layer, for which the damage to the substrates by light energy can be neglected.

Because the drying period is relatively short, the R2R intense pulsed light compatible method can realize short-term ambient condition R2R fabrication, which reduces the cost of additional shield-gas and is considered to be a low-cost method suitable for industrial application.

\section{Discussion and outlook on R2R technique advancements}

Towards the need for a low-cost, high-efficient PSCs industrial production, a high PCE of marketing PSCs should be firstly achieved. To further enhance the price competitiveness of PSCs devices, a flow line production pattern under an ambient condition should be developed.

\subsection{Improvements on the perovskite morphology and grain scale for better PCE}

The morphology quality of PSC layers is the crucial factor which will influence cell PCE. PSCs with well-uniformed, less-defected layer and a better morphology are experimented to have relatively higher PCE and more stable property[22]. The inject-printing, blade-coating or slot-die coating R2R compatible methods can achieve high-quality layer by precisely controlled coating parameters. Proper heat treatment on perovskite layer as annealing process or gas-quenching process can also aid in forming a uniform layer[20]. It is well noticed that an optimized layer morphology required by Hwang et al. was in cloudy form instead of a dense form, which accelerates the reaction between perovskite precursors. (Toward Large Scale Roll-to-Roll Production of Fully Printed Perovskite Solar Cells)

Large scale crystalline perovskite grains enable higher cell performance than small-grain perovskite PSCs[2]. The perovskite layer with proper heat treatment is observed as an obvious grain growth[12,20]. And a $\mathrm{MACl}$ addition into the perovskite precursor can induce the secondary crystalline grain growth and reduce pin-hole defects, which shows better PCE performance[14].

\subsection{Natural ambient condition for R2R production}

The industrial production of PSCs seeks a low-cost fabrication method for more marketing competitiveness. Current laboratorial methods are commonly conducted in an arid environment. However, industrial-scale productions require high input to maintain drying condition, which reduces the cost-efficiency of PSCs. Under a natural ambient condition, reducing the sintering period is shown as an adaptive method to impede environmental moisture erosion. A high-temperature sintering technique or intensified light drying method can achieve short-time processing[20]. 


\subsection{Enhancements in Moisture resistance of PSCs}

Product stability is an essential factor in product quality. Organic lead halide perovskite, as a common component of PCS device, is of moisture irresistance[23,24]. Currently there exists two major principles in enhancing moisture resistance, one principle is adding poly-additives into the perovskite precursor as protection from moisture erosion, another is improving hole transport layer materials.

Zhao et al. applied polyethylene glycol (PEG) as a long-chain insulating poly-additive into perovskite precursor. The moisture-resisting performance of poly-additive PCE was enhanced. PEG owns excellent hygroscopicity property, which can well protected perovskite layer as a composite reinforcement[25]. Kim et al. used poly(ethylene oxide) (PEO) polymer additive during perovskite crystallizing, and a higher humidity-tolerant PSC was obtained[12].

As for hole layer enhancement, Wei et al. added PEG polymer chain into $\mathrm{SnO} 2$ precursor solution, which forms a stable $\mathrm{SnO} 2-\mathrm{PEG}$ composite. $\mathrm{SnO} 2$ was in the protection of $\mathrm{PEG}$, which enables improved humidity stability of $\mathrm{SnO} 2$ hole transport layer[26]. Jeong et al. substituted traditional Spiro-OMeTAD hole transport material with Spiro-mF and Spiro-oF, and achieved a long endurance under highly humid condition[8].

\section{Outlook over the application of flexible PSCs}

The industrial-produced flexible perovskite solar cells by $\mathrm{R} 2 \mathrm{R}$ method is regarded as an essential supplement on solar cell market, which owns wide applications in flexible device markets compared to rigid Si solar cells.

\subsection{Conception of expecting applications of flexible PSCs}

The flexibility of PSCs is an outstanding feather for extensive application, for which flexible PSCs can be well adaptive onto the curve substrates, and easy for installing.

Commercial flexible PSCs can be packaged in scrolls for convenient transportation and stockpiled. Flexible PSCs which can be installed for contemporary circumstances, such as field-trip tents or movable house. Under such circumstances, flexible PSCs scrolls can be unfolded and installed on open field as power supplement.

On office scenario, flexible PSCs can be packaged in scrolls and installed in the peripheral of office building, which functions as roll-up solar cells in substitute of traditional curtains. When the office building receives a high exposure to daylight, PSCs can be unfolded as effective daylight absorber, which serves as self-cooling shield from sunlight heat and solar cells for electricity generation. When the building requires a sufficient sunlight supplement, flexible PSCs can be reset and package into scrolls, which enable sunlight flowing in.

\subsection{Flexible PSCs combining wind-flutter structural}

Flexible PSCs can combine with a wind-flutter structural energy collector as a hybrid energy collector. The wind-flutter structure, as schematic shown in figure 6 , consists a flexible plastic flutter membrane, which can convert wind-induced vibration energy into electricity $[27,28]$.

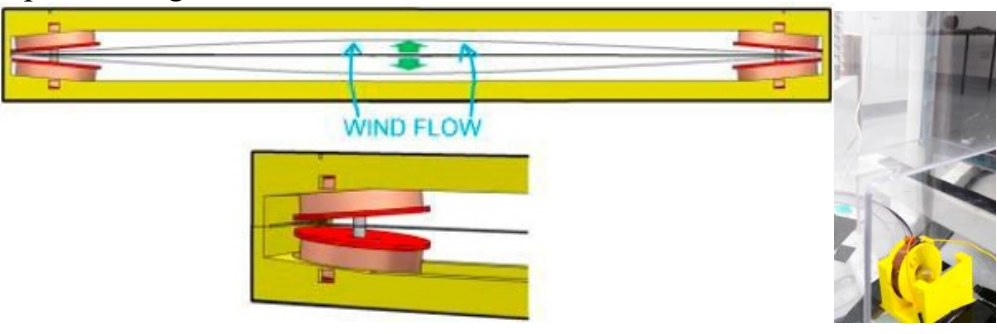

Fig 6. Schematic of wind-flutter structure and tensioned membrane [28]

A perovskite coated wind flutter is proposed to substitute the common membrane with flexible perovskite coated flexible plastic belt. The perovskite can be coated onto the wind belt surface as a flexible solar cell module, while wind belt structure can collect wind energy. Flexible PSC module can serve as a wind belt to harvest wind-energy, which is especially necessary for light-insufficient area or at night. However, perovskite layer should have excellent bending properties and high vibratory fatigue to realize a fully-functioned hybrid energy collector.

Meanwhile, PSCs combined wind flutters devices can be exploited as multi-functional sensor. While wind flutter can serve as sensitive wind sensor, perovskite module can function as photon detector for ultraviolet detector.

\section{Conclusion}

Based on the previous discussion on the R2R compatible methods and the improving prospects, current R2R fabrication methods still have certain technical gap towards industrial applications. As further advancing laboratorial methods realize technique transformation into industrial application, achieving applicable industrial solar cell $\mathrm{R} 2 \mathrm{R}$ productions will be a promising future. It can be optimistically estimated that the solar cell market will have notable boosts as cost-effective PSCs by industrial production were introduced, and 
low-cost accessible PSCs will catalysis the enthusiasm towards solar cells installing as recyclable green energy sources.

The author thanks Olivia Sun for the comments and guidance in this manuscript. Comments and advice on this paper are sincerely appreciated.

\section{References}

1. Galagan Y, Coenen E W C, Verhees W J H , et al. Towards the scaling up of perovskite solar cells and modules[J]. Journal of Materials Chemistry A, 2016, 4(15):5700-5705.

2. Schmidt $T$ M, Larsen-Olsen $T$ T, Jon $E$. CarléDechan Angmo, et al. Upscaling of Perovskite Solar Cells: Fully Ambient Roll Processing of Flexible Perovskite Solar Cells with Printed Back Electrodes[J]. Advanced Energy Materials, 2015, 5(15).

3. Razza S, Castro-Hermosa S, Di Carlo A, et al. Research Update: Large-area deposition, coating, printing, and processing techniques for the upscaling of perovskite solar cell technology[J]. Apl Materials, 2016, 4(9):104003.

4. Bishop J E, Read C D, Smith J A, et al. Fully Spray-Coated Triple-Cation Perovskite Solar Cells[J]. Scientific Reports, 2020, 10(1).

5. Nrel. Best Research-cell Efficiencies 1975-2020[Z]. https://www.nrel.gov/pv/cell-efficiency.html.

6. Mescher H, Schackmar F, Eggers H, et al. Flexible Inkjet-Printed Triple Cation Perovskite X-ray Detectors[J]. ACS Applied Materials \& Interfaces, 2020, 12(13).

7. Zhanhua, Wei, Haining D, et al. Inkjet Printing and Instant Chemical Transformation of a $\mathrm{CH} 3 \mathrm{NH} 3 \mathrm{PbI}$ 3/Nanocarbon Electrode and Interface for Planar Perovskite Solar Cells[J]. Angewandte Chemie, 2014, 126(48):13455-13459.

8. Jeong M, Choi I W, Go E M, et al. Stable perovskite solar cells with efficiency exceeding $24.8 \%$ and $0.3-\mathrm{V}$ voltage loss[J]. Science, 369.

9. Kim $\mathrm{Y}$ Y, Yang $\mathrm{T} \mathrm{Y}$, Suhonen $\mathrm{R}$, et al. Gravure-Printed Flexible Perovskite Solar Cells: Toward Roll-to-Roll Manufacturing[J]. Advanced Science, 2019.

10. Kyeongil, Hwang, Yen-Sook, et al. Toward large scale roll-to-roll production of fully printed perovskite solar cells.[J]. Advanced materials (Deerfield Beach, Fla.), 2015.

11. Sears K K, Fievez M, Gao M, et al. ITO Free Flexible Perovskite Solar Cells Based on Roll-to-Roll, Slot-die Coated Silver Nanowire Electrodes[J]. Solar RRL, 2017,1(8).

12. Kim, Jueng-Eun, Kim, Seok-Soon, Zuo C, et al. Humidity-Tolerant Roll-to-Roll Fabrication of Perovskite Solar Cells via Polymer-Additive-Assisted Hot Slot Die
Deposition[J]. Advanced Functional Materials, 2019, 29(26):1809194.1-1809194.9.

13. Deng Y, Peng E, Shao Y, et al. Scalable fabrication of efficient organolead trihalide perovskite solar cells with doctor-bladed active layers[J]. Energy \& Environmental Science, 2015, 8(5):1544-1550.

14. Tang S, Deng Y, Zheng X, et al. Composition Engineering in Doctor - Blading of Perovskite Solar Cells[J]. Advanced Energy Materials, 2017, 7(18):1700302.1-1700302.7.

15. Mathies F, Abzieher T, Hochstuhl A, et al. Multipass inkjet printed planar methylammonium lead iodide perovskite solar cells[J]. Journal of Materials Chemistry A, 2016:10.1039.C6TA07972E.

16. OLED-inform. OLED ink jet printing: introduction and market status[EB/OL]

https://www.oled-info.com/oled-inkjet-printing.

17. Zhanhua, Wei, Haining D, et al. Inkjet Printing and Instant Chemical Transformation of a $\mathrm{CH} 3 \mathrm{NH} 3 \mathrm{PbI}$ /Nanocarbon Electrode and Interface for Planar Perovskite Solar Cells[J]. Angewandte Chemie, 2014, 126(48):13455-13459.

18. Haotong, Wei, Jinsong, et al. Halide lead perovskites for ionizing radiation detection.[J]. Nature communications, 2019.

19. Liu $\mathrm{X} \mathrm{K}, \mathrm{Xu} \mathrm{W}$, Bai $\mathrm{S}$, et al. Metal halide perovskites for light-emitting diodes[J]. Nature Materials, 2020.

20. Druffel T, Dharmadasa R, Lavery B W, et al. Intense pulsed light processing for photovoltaic manufacturing[J]. Solar Energy Materials \& Solar Cells, 2018, 174:359-369.

21. A. H. Ghahremani and T. Druffel, Realizing Perovskite Solar Cells on Roll Roll-to-Roll Compatible Processes, 2019 IEEE 46th Photovoltaic Specialists Conference (PVSC), Chicago, IL, USA, 2019, pp. 1-5, doi: 10.1109/PVSC40753.2019.9198957.

22. Kim Y Y, Yang T Y, Suhonen R, et al. Roll-to-roll gravure-printed flexible perovskite solar cells using eco-friendly antisolvent bathing with wide processing window $[\mathrm{J}]$. Nature Communications.

23. Wu, M., Haji Ladi, N., Yi, Z., Li, H., Shen, Y. and Wang, M. (2020), Stability Issue of Perovskite Solar Cells under Real - World Operating Conditions. Energy Technol., 8: 1900744.

24. Di J, Chang J, Liu S F. Recent progress of two-imensional lead halide perovskite single crystals: Crystal growth, physical properties, and device applications[J]. EcoMat, 2020.

25. Zhao Y, Wei J, Li H, et al. A polymer scaffold for self-healing perovskite solar cells[J]. Nature Communications, 2016, 7:10228.

26. SnO2-in-Polymer Matrix for High-Efficiency Perovskite Solar Cells with Improved Reproducibility and Stability. 
27. Fei F, Mai J D, Li W J. A wind-flutter energy converter for powering wireless sensors $[\mathrm{J}]$. Sensors \& Actuators A Physical, 2012, 173(1):163-171.
28. Aquino A I, Calautit J K, Hughes B R. A Study on the Wind-Induced Flutter Energy Harvester (WIFEH) Integration into Buildings[J]. Energy Procedia, 2017, 142:321-327 